\title{
Estimation of Polyvinyl Alcohol Cryogel Mechanical Properties with Four Ultrasound Elastography Methods and Comparison with Gold Standard Testings
}

\author{
Jérémie Fromageau, Jean-Luc Gennisson, Cédric Schmitt, Roch L. Maurice, Rosaire Mongrain, \\ and Guy Cloutier, Member, IEEE
}

\begin{abstract}
Tissue-mimicking phantoms are very useful in the field of tissue characterization and essential in elastography for the purpose of validating motion estimators. This study is dedicated to the characterization of polyvinyl alcohol cryogel (PVA-C) for these types of applications. A strict fabrication procedure was defined to optimize the reproducibility of phantoms having a similar elasticity. Following mechanical stretching tests, the phantoms were used to compare the accuracy of four different elastography methods. The four methods were based on a one-dimensional (1-D) scaling factor estimation, on two different implementations of a 2-D Lagrangian speckle model estimator (quasistatic elastography methods), and on a 1-D shear wave transient elastography technique (dynamic method). Young's modulus was investigated as a function of the number of freeze-thaw cycles of PVA-C, and of the concentration of acoustic scatterers. Other mechanical and acoustic parameters - such as the speed of sound, shear wave velocity, mass density, and Poisson's ratio - also were assessed. The Poisson's ratio was estimated with good precision at 0.499 for all samples, and the Young's moduli varied in a range of $20 \mathrm{kPa}$ for one freeze-thaw cycle to $600 \mathrm{kPa}$ for 10 cycles. Nevertheless, above six freeze-thaw cycles, the results were less reliable because of sample geometry artifacts. However, for the samples that underwent less than seven freeze-thaw cycles, the Young's moduli estimated with the four elastography methods showed good matching with the mechanical tensile tests with a regression coefficient varying from 0.97 to 1.07 , and correlations $R^{2}$ varying from 0.93 to 0.99 , depending on the method.
\end{abstract}

Manuscript received December 12, 2005; accepted November 7, 2006. The financial support for this research was provided by Valorisation-Recherche Québec (group grant \#2200-094) and the Natural Sciences and Engineering Research Council of Canada (grant \#138570-01). Dr. Cloutier is recipient of the National Scientist award of the Fonds de la Recherche en Santé du Québec (FRSQ, 20042009), Dr. Maurice holds a Research Scholarship award of FRSQ, and Dr. Fromageau received a postdoctoral studentship award from the Groupe de Recherche en Sciences et Technologies Biomédicales of the Institute of Biomedical Engineering of the École Polytechnique and University of Montreal.

J. Fromageau, J.-L. Gennisson, C. Schmitt, R. L. Maurice, and G. Cloutier are with the Laboratory of Biorheology and Medical Ultrasonics, University of Montreal Hospital Research Center, Montréal, Québec, H2L 2W5, Canada (e-mail: guy.cloutier@umontreal.ca).

R. Mongrain is with the Mechanical Engineering Department, McGill University, Montréal, Québec, H3A 2K6, Canada.

Digital Object Identifier 10.1109/TUFFC.2007.273

\section{INTRODUCTION}

$\mathrm{P}$ OLYVINYL alcohol cryogels (PVA-C) are polymers that become harder with an increase in the number of freeze-thaw cycles. Their potential in biotechnology and medicine is manifold [1], notably for building biological tissue-mimicking phantoms. For phantom designs, this material presents the advantage of being compatible to both magnetic resonance and ultrasound imaging. Nevertheless, the literature indicates that the physical properties depend on possible dehydration during heating at the first step of preparation, the speed of decreasing and increasing temperatures, the minimum temperature reached, the volume of the sample, and the number of freeze-thaw cycles [1] [4]. All these parameters are hardly reproducible in time, and the elasticity can be considered as a nondeterminist value. In this paper, we devoted undivided attention to the preparation stage.

Beside PVA-C, other multimodality materials mimicking biological tissues have been described in the literature, especially water-based gels that are the most used [5]-[8]. Compared to these materials, cryogel phantoms are simple to prepare because gelatin-based phantoms need aldehydes linking to have a long life time. Another PVA big advantage is its biocompatibility. For instance, PVA gels are hydrophilic and likely capable of protein absorption, thus supporting cellular growth [2].

Different methods have been developed to provide imaging of the elastic properties of biological tissues. Static elastography methods are motion estimators, which use sequences of images acquired at different levels of compression/dilation to extract tissue mechanical parameters such as tissue displacements, strains, shears, and elastic moduli. Motion estimators described in the literature are based on cross-correlation algorithms [9], [10], on different implementations of a Lagrangian speckle model [11][13], on spectral techniques [14], [15], or on a zero-crossing method [16]. Strain can be estimated by several methods; most of the published techniques assess a displacement field and deduce the axial strain by a derivative operation [10], [17]-[19], whereas other methods directly estimate the axial strain as a time-scaling factor [20], [21] or by estimating every component of the strain tensor [12]. On the other hand, transient elastography is a dynamic method that es- 
timates mechanical properties by measuring the speed of shear waves propagating in tissues. Shear wave speed is directly related to Young's modulus [22], [23]. With this method, the medium is excited with a low-frequency pulse, allowing the propagation of a bulk wave and a shear wave. Shear wave speed can be estimated along an A-line, with a cross-correlation algorithm.

In this paper, the Young's modulus and the reproducibility of PVA samples were evaluated with a mechanical test instrument as a gold-standard reference. The results then were compared to those estimated with four different elastography methods. A dynamic elastography technique [24] and three quasistatic elastography methods were used. These last three methods were based on a scaling factor estimation [25] and on two different implementations of the Lagrangian speckle model [26], [27]. In Section II, the experimental setup used to prepare PVA is described. Following that, the four methods used to measure Young's moduli are reported, experimental setups are presented, and the four algorithms are introduced. The results are summarized in Section III. Section IV is dedicated to the discussion about reproducibility over samples, techniques of measurement, and the behavior of samples according to the preparation variables. Section V draws a brief conclusion.

\section{Materials AND Methods}

\section{A. PVA Sample Preparation}

A rigorous preparation process was defined. The solution used had a concentration of $10 \%$ by weight of polyvinyl alcohol dissolved in pure water (CAS 7732-185) and ethenol homopolymer (CAS 9002-89-5), as indicated by the manufacturer. The solutions came from the same batch for the entire experiments (lot \#407101, Beacon, NY). The solution container was heated in hot water to $80^{\circ} \mathrm{C}$ and, to minimize dehydration, the container was covered. When the mixture was fluid, it was mixed with Sigmacell particles (Sigmacell Cellulose, type 20, Sigma Chemical, St. Louis, MO). The Sigmacell particles served as acoustic scatterers to allow good ultrasonic signals; the average particle size was $20 \mu \mathrm{m}$. The weight by weight percentage of added Sigmacell varied from 1\% to $4 \%$ to study the impact of this variable on mechanical properties. Solidification and polymerization of PVA samples were induced by freezing-thawing cycles (from 1 to 10 cycles) in a temperature-controlled chamber. The specifically designed chamber was composed of a freezer equipped with heated elements (type YF-204017, Supra Scientifique, Terrebonne, QC, Canada) and of an electronic controller (Model 981, Watlow, Winona, MN), which allowed temperature regulation. As the processing conditions of the freeze-thaw cycles play a major role on final mechanical properties [2], they were carefully chosen. A freeze-thaw cycle lasted 24 hours, and the freeze-thaw rate (the slope of increase or decrease in temperature) was $\pm 0.2^{\circ} \mathrm{C}$ /minute.
The maximum and minimum temperatures were $20^{\circ} \mathrm{C}$ and $-20^{\circ} \mathrm{C}$, respectively. A cycle then was constituted of two holding stages of 8 hours 40 minutes at $+20^{\circ} \mathrm{C}$ and $-20^{\circ} \mathrm{C}$, and two periods of 3 hours 20 minutes when the temperature changed from one extremum to the other. At the end of the last cycle, the samples were cut to obtain flat surfaces and put in water at room temperature.

Two series of samples were tested. For one series, the number of freeze-thaw cycles varied. To assess operator reproducibility, three different operators prepared 10 samples each (from 1 to 10 cycles), with $3 \%$ of Sigmacell as acoustic scatterers. In the second series, the percentage of Sigmacell varied. Eight samples with two freeze-thaw cycles were built by one operator with an increasing ratio of Sigmacell (two samples for 1, 2, 3, and 4\% of Sigmacell). The samples were poured into cylindrical moulds with a $60-\mathrm{mm}$ diameter and cut to a height of $20 \mathrm{~mm}$. All experiments were performed on the same phantoms, except the tensile tests. For the latter, small samples were required because the mechanical test instrument is adapted for biological tissues of small size. These last measurements (destructive testing) were performed when all elastography data were analyzed, and core samples of 3-mm diameter were extruded from the center of the whole samples.

\section{B. Density and Speed of Sound Assessments}

Density and speed of sound are two intrinsic and important material parameters of interest for comparison with biological tissues. Furthermore, these parameters are required by some elastography methods to calculate Young's modulus. Their precise measurements are then of considerable interest. Density was measured according to Archimede's principle. Twenty-five batches of known density liquids were prepared at room temperature by mixing a volume of glycerol, $\rho_{\text {gly }}=1250 \mathrm{~kg} / \mathrm{m}^{3}$ (type G-3730, ACP, Montreal, QC, Canada), in a volume of water, $\rho_{\text {wat }}=1000 \mathrm{~kg} / \mathrm{m}^{3}$. Solution densities varied from $1025 \mathrm{~kg} / \mathrm{m}^{3}$ to $1060 \mathrm{~kg} / \mathrm{m}^{3}$. Density was determined by finding the solution in which the sample floated in a midequilibrium position.

Speed of sound was measured in reflection mode, on a plane surface immersed in a bath containing distillated water at $18.7 \pm 0.1^{\circ} \mathrm{C}$, with an ultrasonic wave emitted by a single-element $5 \mathrm{MHz}$ central frequency transducer (Model V310, Panametrics, Waltham, MA). The time shift between a reference echo acquired in distilled water, and an echo acquired for each PVA sample, gave the ratio between the speed of sound in water and that in PVA. The speed of sound $c$ in each specimen was measured by:

$$
c=v_{w}(T) \frac{\tau-t_{2}}{t_{1}-t_{2}}
$$

where $v_{w}(T)$ is the speed of sound in distilled water, which depends on the temperature (T), $\tau$ is the time of flight of the reference echo from the flat reflector, $t_{1}$ is the time of flight of the echo from the reflector in the presence of PVA, and $t_{2}$ is the time of flight of the first PVA's interface 
echo. Then $\left(t_{1}-t_{2}\right)$ corresponds to the wave's time of flight to cross the PVA sample and $\left(\tau-t_{2}\right)$ corresponds to the wave's time of flight to cross the same distance in distilled water. To improve reliability, the speed of sound in each PVA sample was averaged over 100 acquired signals.

\section{Quasi-Static Elastography}

Static elastography measurements were performed with a homemade instrument, allowing compression and relaxation of samples, and simultaneous acquisitions of radio frequency (RF) ultrasound data. The stress data were recorded from the homemade instrument. With this setup, the stress in the sample was calculated as the ratio of weight over the sample section under resting condition. We limited the region of interest to the center of each sample, in a region covering about $50 \%$ of the sample diameter. In this region, the stress was considered uniform, and the sample surface was considered constant during loading. Axial strains were estimated with the three quasistatic elastography methods described below. Each of these methods could provide an image of local strain distribution (an elastogram) within each sample. The mean strain value was extracted for each elastogram, and the cumulative sum over all compression stages was used to plot typical stress-strain curves. Knowing the stress and strain relationships, the Young's moduli were measured as the slope of the linear portion of the stress-strain curves (strains $<7 \%$ ), for the load phase (i.e., during compression).

The homemade mechanical test instrument was composed of a stepper motor (Compumotor Zeta E57, Parker Hannifin Corporation, Rohnert Park, CA), driven by a controller (Model Zeta 6104, Parker Hannifin Corporation), commanding the compressor displacements. The load on each PVA sample was measured as the weight on a precision balance (Model TS4KS, Ohaus Corporation, Florham Park, NJ). During the whole dynamic compression and relaxation of a sample, the load was continuously monitored and sent to a personal computer via an acquisition card for off-line processing (CS8500 PCI, National Instruments, Austin, TX). Before recording RF data, a preconditioning phase of one compression-dilation cycle at the speed of $0.45 \mathrm{~mm} / \mathrm{s}$ was imposed to the PVA samples. The compression plate was made of Plexiglas and had a size larger than the sample diameter, to impose a uniform stress condition. The ultrasound RF signals were recorded through a hole made in the plate of Plexiglas in which the probe was positioned. As the probe fitted perfectly into the hole, there was no problem of stress discontinuity. Stress curves were timely registered, in postprocessing, with cumulated strain curves by fitting the maxima and minima of the load phase.

Ultrasonic acquisitions were performed with a computerbased clinical instrument providing RF data (Model 500RP, Ultrasonix Medical Corp., Burnaby, BC, Canada). It was equipped with a 128 -elements ultrasonic linear array (Type L12-5, Ultrasonix Medical Corp.) of $6.6 \mathrm{MHz}$ cen- tral frequency. RF images provided through the research package had $254 \mathrm{RF}$ lines; they were digitized with a sampling frequency of $40 \mathrm{MHz}$, and the frame rate was of 15 images/s. RF data were acquired continuously during the compression and relaxation stages.

As mentioned earlier, the local strain was estimated by three different quasistatic elastography methods. Each coauthor applied his own method, with respective choices of the 1-D or 2-D window size and RF image increment. The choice of these parameters, described below, was made blindly (the Young's moduli were unknown to each coinvestigator as they were the last measures collected), and it was performed to optimize the elastograms according to each author's perception.

1. Time-Scaling Factor Algorithm: The first quasistatic elastography algorithm is based on a 1-D cross-correlation technique. The parameter used to evaluate the strain is the time-scaling factor between RF signals. The assumption is that, if a tissue undergoes a strain $\varepsilon$, then the ultrasound $\mathrm{RF}$ signal scattered by the tissue after deformation undergoes the same time-scaling factor $\varepsilon$. The RF signal after deformation $s(t)$ can be written as a function of the RF signal before deformation $r(t)$ as:

$$
s(t)=r(t(1+\varepsilon))
$$

To estimate the time-scaling factor, the ambiguity function $R_{\check{r} s}(\eta)$ with a constant zero delay was calculated as:

$$
R_{\check{r} s}(\eta)=\int_{0}^{T} \check{r}(t) s(t(1-\eta)) d t,
$$

where $\check{r}(t)$ is the Hilbert transform of $r(t)$, and $T$ is the size of the calculation window. Fromageau et al. [25] have shown that it is possible to estimate the time-scaling factor from the unique value $\boldsymbol{R}_{\check{r} s}(0)$ with the following relationship:

$$
\varepsilon=\frac{1}{\pi f_{0} T} \arcsin \left(\frac{\boldsymbol{R}_{\check{r} s}(0)}{E_{s}}\right),
$$

with $f_{0}$ being the central frequency of the RF signals, and $E_{s}$ the energy of the signal $s(t)$ over the sampling window $T$. A strain image was obtained by applying the 1-D estimator on moving windows across each of the $254 \mathrm{RF}$ line of the image. The window length was about 15 wavelengths; that is to say, 100 pixels and the overlap was 98 pixels.

As the ratio between the ultrasonic frame rate and the stepper motor compression speed was high, the compression between two consecutive RF images was small $(<0.2 \%$ strain). Because the variance of most estimators is generally reduced by considering larger strains [28], the deformations were estimated from RF images with an interleave of 3 (i.e., we used RF images number $1-4,2-5, \ldots$, $(\mathrm{N}-3)-\mathrm{N})$. To deduce the strain between two consecutive images, the values calculated were divided by 3 . 


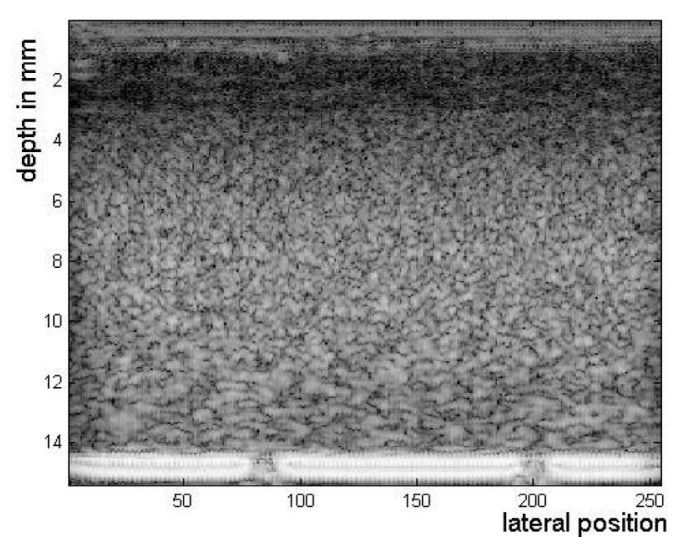

(a)

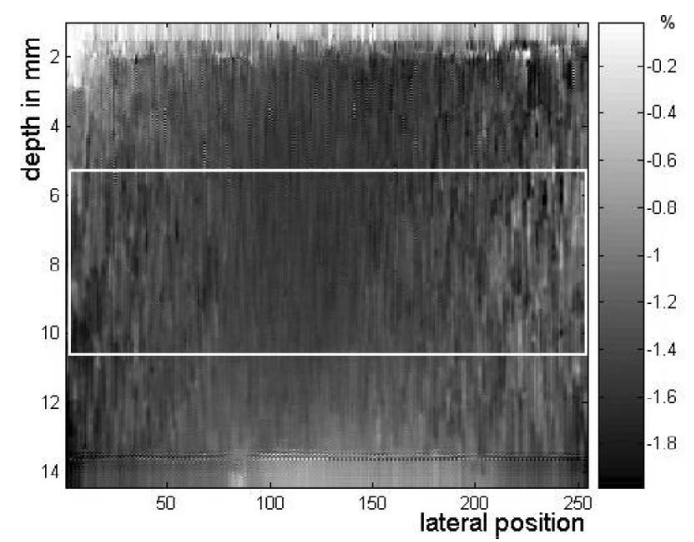

(b)

Fig. 1. Reconstructed envelope B-mode image (a) and corresponding elastogram (b) computed with the time-scaling factor algorithm. For each elastogram, the mean strain value was calculated in the ROI that was centered around the focus depth (white rectangle).

Due to the boundary conditions and to the geometry of PVA samples, it is assumed that the strain was constant over the whole sample. Moreover, because the samples were homogeneous and isotropic, and because of the large number of data to be reported, we limited the analysis to the mean strain value within each PVA sample. The mean deformation was calculated over a window centered on the focal zone of the ultrasound instrument [see Fig. 1(b)]. This window of $180 \times 254$ pixels was located in the middle of the PVA sample to avoid boundary artifacts and regions with a low signal-to-noise ratio (SNR).

2. First Lagrangian Algorithm: The second quasi-static algorithm is a 2-D Lagrangian speckle model estimator (LSME). This method was described in details previously [11], [26]. It requires partitioning the RF images into small regions of interest (ROI), in which tissue motion is assumed to be affine. The translation part having been compensated for appropriately with a cross-correlation technique, the linear part given as a 2-D linear transformation matrix (LT), can be related to the strain tensor through the following relationship:

$$
\begin{aligned}
\varepsilon_{i j}(t) & =\frac{1}{2}\left[\Delta_{i j}(t)+\Delta_{j i}(t)\right], \\
\text { with: } \Delta & =L T-[I]=\left(\begin{array}{ll}
\frac{\partial U_{x}}{\partial x} & \frac{\partial U_{x}}{\partial y} \\
\frac{\partial U_{y}}{\partial x} & \frac{\partial U_{y}}{\partial y}
\end{array}\right) .
\end{aligned}
$$

In this equation, $U_{x}$ and $U_{y}$ are the lateral and axial displacement fields, respectively. $\Delta$ is defined as the deformation matrix. The maps of $\Delta_{22}\left(=\varepsilon_{22}\right)$, known as the axial strain, provided the elastograms shown in the current study. [I] is the 2-D identity matrix. The LSME, for a given ROI, can mathematically be formulated as the following nonlinear minimization problem:

$$
\underset{\mathrm{LT}}{\mathrm{MIN}}\left\|I(x, y, t)-I_{\mathrm{Lag}}(x, y, t+\Delta t)\right\|^{2},
$$

where $(x, y)$ defines the image coordinate system, and $t$ indicates time. $I(x, y, t)$ is the pretissue-motion $\mathrm{RF}$ image, and $I_{\mathrm{Lag}}(x, y, t+\Delta t)$ is the Lagrangian speckle image
(LSI) at time $t+\Delta t$. It is worth mentioning that the LSI is defined as a posttissue-motion RF image $I(x, y, t+\Delta t)$ that was numerically compensated for tissue motion, as to achieve the best match with $I(x, y, t)[12]$. The minimum of (6) was obtained by using the appropriate LT; and (6) was solved using the optical flow-based implementation of the LSME developed in [29]. The deformation parameters then were estimated using an inversion algorithm. For the current study, the measurement window required for the LSME algorithm was set to $200 \times 20$ pixels, axially and laterally, respectively (with $90 \%$ and $80 \%$ axial and lateral overlaps). The mean strain values reported here were obtained by averaging the tensor component, $\varepsilon_{22}$, over elastograms computed in the focal zone. In opposition to the time-scaling algorithm described earlier, $\varepsilon_{22}$ was estimated on successive RF images corresponding to strain values below $0.2 \%$.

3. Second Lagrangian Algorithm: The third quasistatic elastography algorithm also is based on the LSME. It is a modified version that considers (5) and (6), and additional parameters to take into account a possible linear intensity variation of the speckle, due to the movement of scatterers regarding the ultrasound field [27], [30]. With this assumption, two coefficients are added in (7). The minimization problem of (6) now becomes (7) (see next page), where $\lambda$, a multiplicative coefficient, represents the contrast of the RF image, $\gamma$, an offset, represents the brightness and $\vec{m}$ is the vector including the motion and intensity variation parameters. The error $E_{\mathrm{ROI}}(\vec{m})$ is minimum when the gradient is zero. This leads to the solution:

$$
\vec{m}=\left[\sum_{x, y \in \mathrm{ROI}} \vec{a} \vec{a}^{T}\right]^{-1}\left[\sum_{x, y \in \mathrm{ROI}} \vec{a} \alpha\right] .
$$

The size of the ROI (measurement windows) of this LSME algorithm was set to $130 \times 50$ pixels, with an overlap of $80 \%$ in both axial and lateral directions $(104 \times 40$ pixels). The axial strain, $\varepsilon_{22}$, was estimated from successive RF images (strain $<0.2 \%$ ), and a mean value was 


$$
\begin{aligned}
E_{\mathrm{ROI}}(\vec{m}) & =\sum_{x, y \in \mathrm{ROI}}[\underbrace{\left(I_{t}-I+x I_{x}+y I_{y}\right)}_{\alpha}-\underbrace{\left(x I_{x} y I_{x} I_{x} x I_{y} y I_{y} I_{y} I 1\right)}_{\vec{a}} \cdot(\underbrace{\Delta_{11} \Delta_{12} d_{x} \Delta_{21} \Delta_{22} d_{y} \lambda \gamma}_{x, y \in \mathrm{ROI}})^{T}]^{2} \\
& =\sum_{\vec{m}}\left[\alpha-\vec{a} \cdot \vec{m}^{T}\right]^{2}
\end{aligned}
$$

obtained by averaging over the whole elastogram. A median filter $(5 \times 5$ pixels $)$ was used to reduce the variance of the estimator.

\section{Transient Elastography}

For the transient elastography assessments, the 1-D shear elasticity probe was used [24]. It was designed with a single-element, 5-MHz ultrasonic transducer (Model V310, Panametrics, Waltham, MA) mounted on a mini-shaker (Type 4810, Brüel\&Kjær, Nærum, Denmark). Shear waves were generated by the front face of the transducer while it was working in a pulse echo mode. Ultrasound longitudinal waves were generated by firing the $5-\mathrm{MHz}$ probe with a pulsed echo system (Model 5900PR, Panametrics), and the low frequency pulse $(200 \mathrm{~Hz})$, producing shear waves, was sent to the mini-shaker with a function generator (Model 33250A, Agilent, Palo Alto, CA) and amplified (Type 2706, Brüel\&Kjær). In a typical experiment, 600 echographic lines were recorded in an 8-bit format with an acquisition card (Compuscope 8500, Gage, Lachine, QC, Canada) on a personal computer at a $100-\mathrm{MHz}$ sampling frequency. The repetition frequency between successive Ascans was fixed in the experiments at $10 \mathrm{kHz}$.

1. Typical Displacement Fields: The ultrasonic signals acquired were compensated off line for the relative motion of the transducer [24]. The longitudinal component of the shear wave displacements along the ultrasonic beam then was computed with a cross-correlation algorithm [9], [10], [31] between successive ultrasonic signals. The displacement image was obtained using a 68 pixels window, with an overlap of 50 pixels. In Fig. 2, displacement fields are shown for different PVA samples with different numbers of freeze-thaw cycles $(1,5$, and 10 cycles). At time $5 \mathrm{~ms}$, a low-frequency $(200 \mathrm{~Hz})$ pulse was given with the front face of the transducer to the PVA sample in order to induce acoustic shear waves. Then, shear waves (quasitransversal waves $\mathrm{S}$ ) were propagated slowly (from $1 \mathrm{~m} / \mathrm{s}$ to $15 \mathrm{~m} / \mathrm{s}$ ) and took a certain time to arrive at each depth. The shear wave slope, plotted on Fig. 2, is related to the shear wave velocity and in the approximation of purely elastic solid, velocity is directly proportional to elasticity [32].

2. Inverse Problem Approach: From the displacement fields, a simple inverse problem approach based on the 1-D Helmholtz equation in a purely elastic medium was taken to recover the shear velocity $\left(V_{S}\right)$ from:
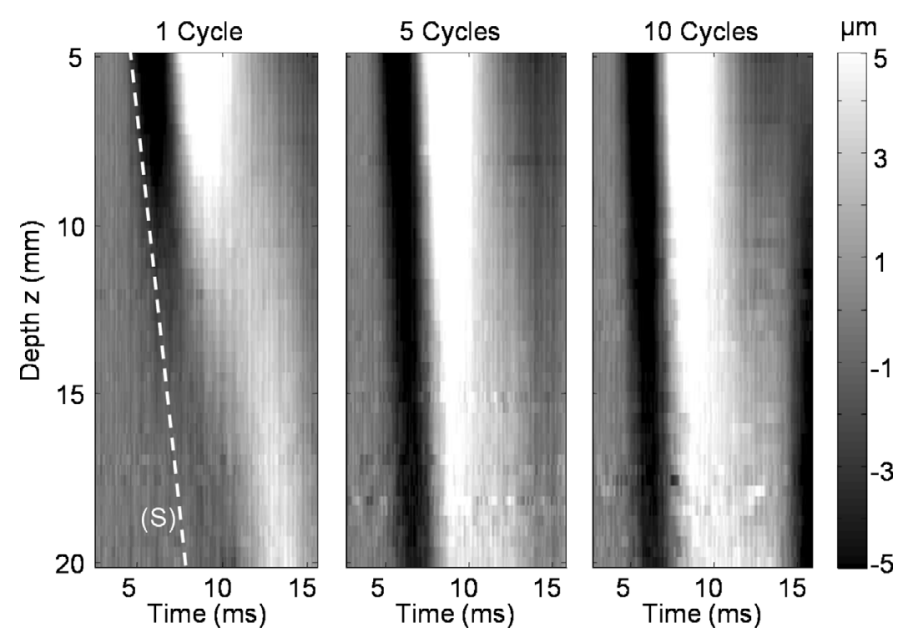

Fig. 2. Displacement field due to the shear wave in three different PVA samples with $3 \%$ of Sigmacell (1, 5, and 10 freeze-thaw cycles). The displacement field was plotted in gray color scale, along depth z as a function of time. The low-frequency pulse at $200 \mathrm{~Hz}$ was given at the time of $5 \mathrm{~ms}$.

$$
\frac{\partial^{2} \mathrm{FT}\left(u_{z}(z)\right)}{\partial z^{2}}-k^{2} \mathrm{FT}\left(u_{z}(z)\right)=0
$$

where FT is the Fourier transform, $u_{z}(z)$ is the longitudinal component of the shear wave displacement field, $z$ is the depth, and $k$ is the wave vector. Thus, the local complex wave vector was given by:

$$
k=\sqrt{\frac{\frac{\partial^{2} \mathrm{FT}\left(u_{z}(z)\right)}{\partial z^{2}}}{\mathrm{FT}\left(u_{z}(z)\right)}},
$$

and the local shear wave velocity $V_{S}[32]$ was obtained with:

$$
V_{S}=\frac{\omega}{\operatorname{Re}[k]},
$$

where $\omega=2 \pi f$ is the pulsation frequency $(f=200 \mathrm{~Hz})$. In practice, derivatives were taken from the displacement image using windows of 20 pixels length and an overlap of 19 pixels.

Now in the general case, acoustic velocities [shear wave velocity $\left(V_{S}\right)$ and speed of sound $(c)$ ] are linked to mechanical properties such as Young's modulus $(E)$, Poisson's ratio $(\nu)$, and density $(\rho)$ [33]. Typically, for an isotropic material, the relationships of these parameters with the sound speed $(c)$ and shear wave speed $\left(V_{s}\right)$ are: 


$$
\begin{gathered}
c=\sqrt{\frac{E(1-\nu)}{\rho(1+\nu)(1-2 \nu)}}, \\
V_{s}=\sqrt{\frac{E}{2 \rho(1+\nu)}} .
\end{gathered}
$$

Accordingly, the Poisson's ratio was retrieved as a function of the two velocities as:

$$
\nu=\frac{c^{2}-2 V_{S}^{2}}{2\left(c^{2}-V_{S}^{2}\right)} .
$$

The Young's modulus was directly retrieved from (13) and, if the material is incompressible, the following equation is acceptable:

$$
E=3 \rho V_{S}^{2}
$$

\section{E. Mechanical Tests (Gold Standard Measures)}

All mechanical tests were done on samples at room temperature. The Young's modulus was measured with a test instrument (ELF 3200, Enduratec, Minnetonka, MN), adapted for biological tissues. The load cell was rated at $225 \mathrm{~N}$ with an accuracy of $0.5 \%$, and the displacement range of the transducer was $12.5 \mathrm{~mm}$ with an accuracy of $0.5 \%$. For these experiments, the machine was programmed to provide a displacement loading.

Tensile testing was performed because it has the advantage of being less sensitive to geometrical boundary imperfection encountered in the building procedure (boundaries do not need to be perfectly flat). Furthermore, isotropic materials have a similar behavior in compression and tension, at least for small deformations. In the present study, the PVA gels were considered isotropic, which is a common assumption [34], [35]. For the stretching test, as mentioned earlier, small core samples were cylindrically cut from the samples used for the elastography tests, and they were fixed between tensile grips. The distance between the grips was $10 \mathrm{~mm}$. Preliminary tests had shown that materials were very stable, then only one cycle was applied during the preconditioning stage. Samples then were subjected to two cycles of a periodic triangular charge. A 6$\mathrm{mm}$ amplitude displacement with a speed of $0.1 \mathrm{~mm} / \mathrm{s}$ was applied to the grips. Young's moduli were calculated, for small strains, in the range of $0-15 \%$ as the slopes of the stress-strain curves obtained, but only on the load section where the behavior is linear. In the following, this method is considered as the gold standard.

\section{RESULTS}

\section{A. Density and Speed of Sound Measurements}

Measurements were performed on PVA samples that underwent from 1 to 10 freeze-thaw cycles with $3 \%$ Sigmacell added. Three samples for each number of cycles

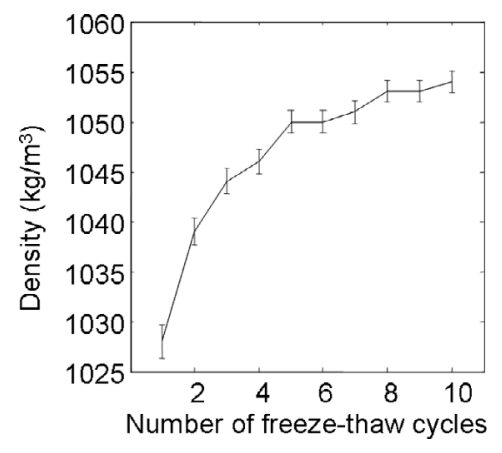

(a)

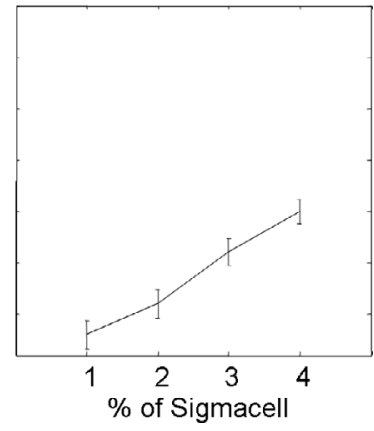

(b)
Fig. 3. Density of PVA samples. (a) As a function of the number of freezing-thawing cycles for $3 \%$ Sigmacell. (b) As a function of the percentage of Sigmacell for two freezing-thawing cycles.

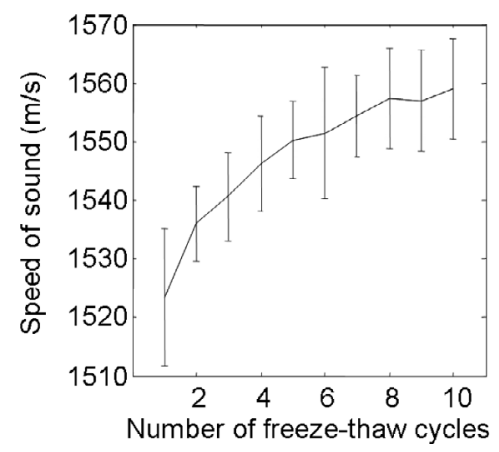

(a)

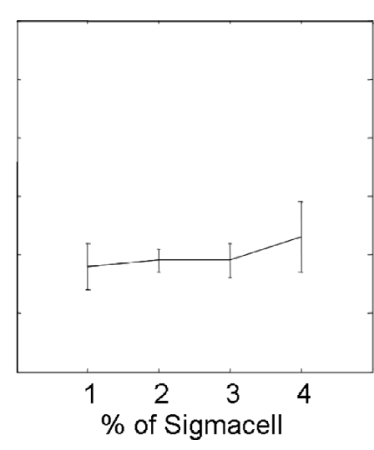

(b)
Fig. 4. Speed of sound in PVA samples. (a) As a function of the number of freezing-thawing cycles for $3 \%$ Sigmacell. (b) As a function of the percentage of Sigmacell for two freezing-thawing cycles.

were built. The mean density as a function of the number of cycles is reported in Fig. 3(a). A logarithmic increasing relationship was observed with a range of variation of the mean densities from 1028 to $1054 \mathrm{~kg} / \mathrm{m}^{3}$. In the second series, which consisted of two samples for each batch, density measurements were performed on samples that underwent two cycles with $1 \%, 2 \%, 3 \%$, and $4 \%$ of Sigmacell added, respectively. The mean density varied linearly between 1028 and $1040 \mathrm{~kg} / \mathrm{m}^{3}$, as shown in Fig. 3(b).

A logarithmic increasing relationship was found in Fig. 4(a) for the speed of sound when the number of freezethaw cycles increased. The values of the speed were in a range between $1525 \mathrm{~ms}^{-1}$ and $1560 \mathrm{~ms}^{-1}$, which is similar to what can be observed in biological tissues. It can be noticed that the concentration of Sigmacell had little impact on the speed of sound [Fig. 4(b)], which remained quite constant at about $1530 \mathrm{~m} / \mathrm{s}$ for concentrations between $1-4 \%$ (two freeze-thaw cycles).

\section{B. Shear Wave Velocity and Poisson's Ratio Assessments}

The shear wave velocity is required to compute the Poisson's ratio, and it was assessed with the transient elastography method. As shown in Fig. 5, a logarithmic increasing relationship was found for this parameter when the 


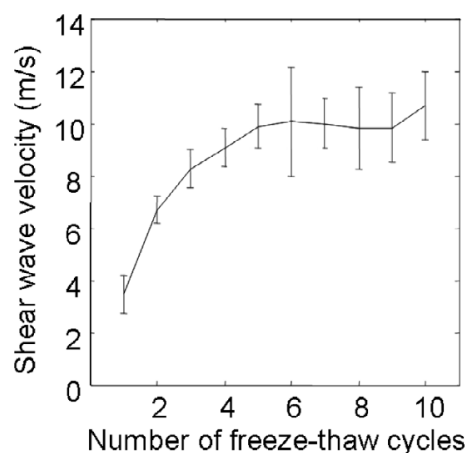

(a)

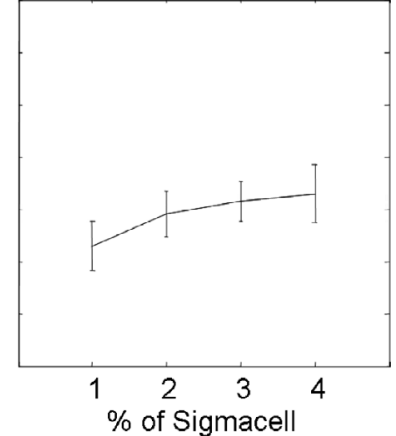

(b)
Fig. 5. Shear wave speed in PVA samples. (a) As a function of the number of freezing-thawing cycles for $3 \%$ Sigmacell. (b) As a function of the percentage of Sigmacell for two freezing-thawing cycles.

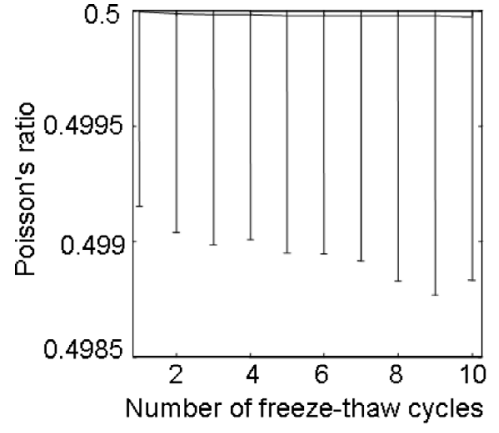

(a)

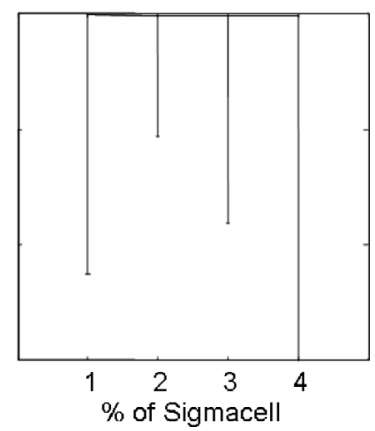

(b)
Fig. 6. Poisson's ratio in PVA samples. (a) As a function of the number of freezing-thawing cycles for 3\% Sigmacell. (b) As a function of the percentage of Sigmacell for two freezing-thawing cycles.

number of freeze-thaw cycles increased. Moreover, it also slightly increased with the concentration of Sigmacell for two freeze-thaw cycles.

With (14), knowing $c$ and $V_{s}$, the Poisson's ratio was estimated as a function of the number of freeze-thaw cycles and of the concentration of Sigmacell. The results are plotted in Fig. 6. As $V_{s}$ is much smaller than $c$, as in every soft biological material [36], our results led to Poisson's ratios close to 0.5 ; all samples had a deviation from 0.5 smaller than $10^{-4}$. Even if a slight decrease was observed when the number of cycles increased, PVA cryogel can be considered as an incompressible material. This result justifies the incompressible assumption made when applying the relationship $E=3 \rho V_{S}^{2}$ to calculate the Young's modulus described below.

\section{Reproducibility of the Method Used to Fabricate PVA Samples}

We also validated the reproducibility of the mechanical properties of PVA. Young's moduli were measured in stretching by the Enduratec instrument that is the gold standard. The results are plotted in Fig. 7 as the bias of each measure in comparison to the mean value calculated over the three samples. The solid lines correspond to the

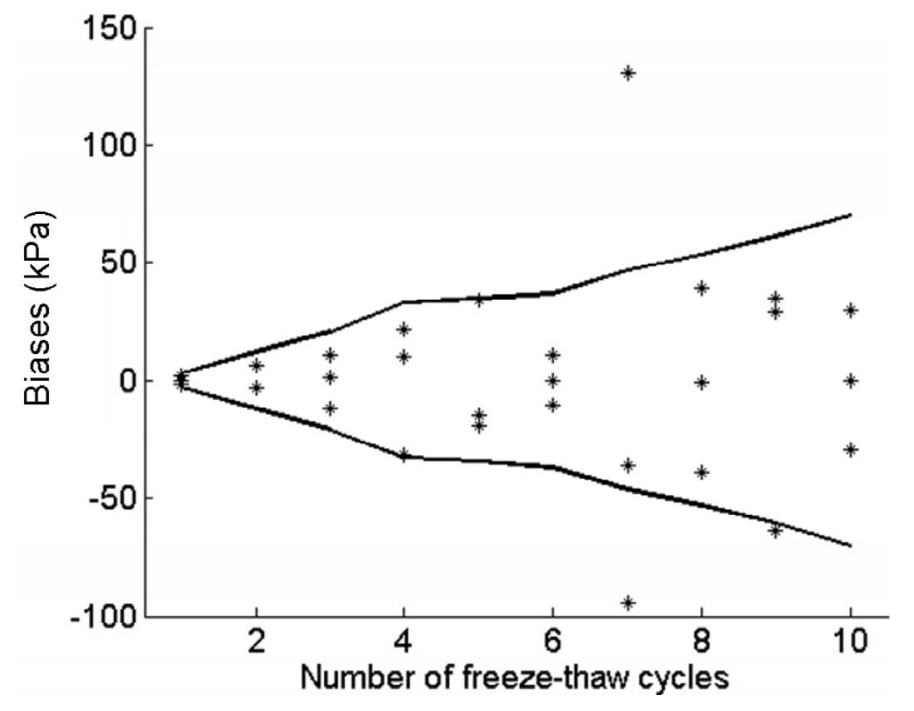

Fig. 7. Young's moduli estimated with the gold standard method on different sets of PVA samples as a function of the number of freezethaw cycles (the Sigmacell concentration was 3\%). The solid lines correspond to the precision interval of the gold standard. Each $*$ corresponds to the bias between a specific measure and the mean. The results are considered reproducible as the three data sets of a same number of cycles are generally within the confidence interval.

precision interval due to sample geometry differences and to the stretching method. $\Delta E / E$ was estimated at about $10 \%$. The results are considered reproducible as the three data sets for a given number of cycles generally lay within the confidence interval.

\section{Young's Modulus Measurement by Different Elastography Methods}

To test the accuracy of the elastography methods, the mean Young's modulus was estimated as a function of the number of freeze-thaw cycles (the Sigmacell concentration was fixed to $3 \%$ ). For each method, the values reported in Fig. 8 correspond to the mean Young's moduli estimated on three different PVA samples built separately. These results are reported with more details in Table I. The standard deviation takes into account the error of each method and of the set of data (fabrication of three different samples).

A first remark is that the Young's moduli assessed with the elastography methods showed a similar trend as the tensile test when the number of cycles was increased (except for 9 and 10 cycles). It is to note that the spreading of the Young's moduli among the different methods was more important for a number of freeze-thaw cycles above six. It is interesting to notice that the scaling factor estimation and the second Lagrangian algorithm provided very similar results for most tests. The first Lagrangian algorithm had a tendency toward an overestimation of Young's moduli for one to six freeze-thaw cycles. The transient elastography method overestimated the rigidity of the samples between one to three cycles, but it provided similar or underestimated Young's moduli for higher numbers of freeze-thaw 
TABLE I

Mean Young's Moduli ( \pm 1 Standard Deviation) Estimated with Each Method for Several Numbers of Freeze-Thaw Cycles.

\begin{tabular}{cccccc}
\hline \multirow{2}{*}{$\begin{array}{c}\text { Number } \\
\text { of cycles }\end{array}$} & Transient & Lagrangian 1 & Lagrangian 2 & Scaling factor & Tensile \\
\cline { 2 - 6 } & $37 \pm 8$ & $39 \pm 1$ & $29 \pm 1$ & $29 \pm 4$ & $25 \pm 3$ \\
1 & $140 \pm 5$ & $132 \pm 4$ & $105 \pm 2$ & $103 \pm 11$ & $105 \pm 12$ \\
3 & $214 \pm 22$ & $206 \pm 14$ & $173 \pm 4$ & $165 \pm 27$ & $182 \pm 21$ \\
4 & $259 \pm 33$ & $300 \pm 25$ & $263 \pm 6$ & $254 \pm 36$ & $286 \pm 33$ \\
5 & $308 \pm 23$ & $357 \pm 41$ & $319 \pm 7$ & $308 \pm 44$ & $302 \pm 35$ \\
6 & $321 \pm 69$ & $358 \pm 42$ & $343 \pm 9$ & $326 \pm 42$ & $322 \pm 37$ \\
7 & $316 \pm 34$ & $411 \pm 29$ & $407 \pm 13$ & $385 \pm 60$ & $398 \pm 46$ \\
8 & $307 \pm 76$ & $437 \pm 35$ & $451 \pm 15$ & $436 \pm 83$ & $465 \pm 53$ \\
9 & $306 \pm 29$ & $381 \pm 20$ & $412 \pm 17$ & $398 \pm 99$ & $532 \pm 61$ \\
10 & $361 \pm 20$ & $458 \pm 18$ & $458 \pm 18$ & $434 \pm 107$ & $615 \pm 70$ \\
\hline
\end{tabular}

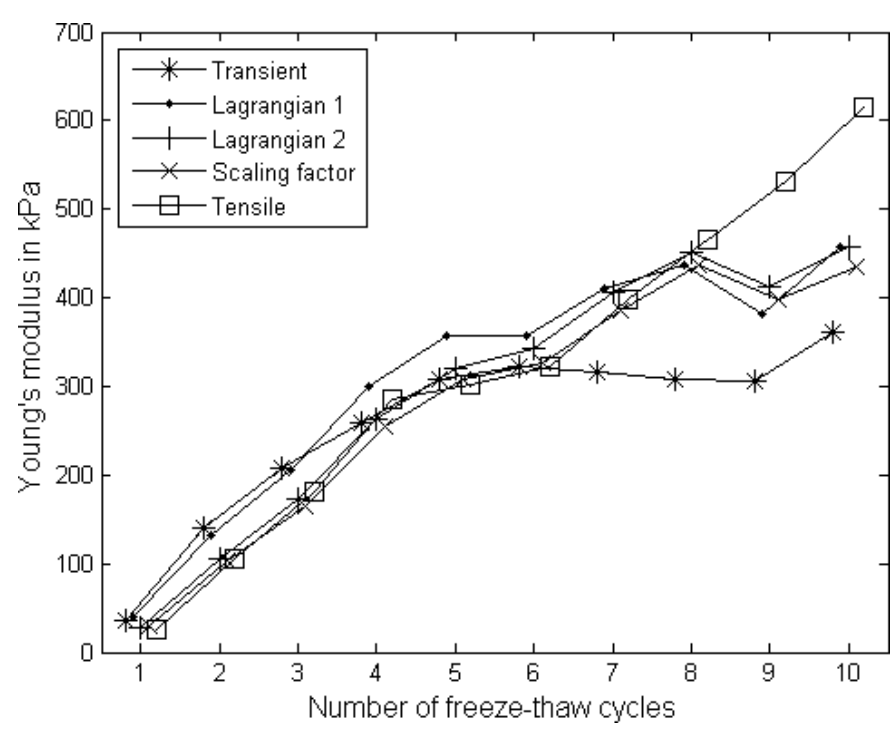

Fig. 8. Mean Young's moduli estimated by different methods as a function of the number of freezing-thawing cycles. A $3 \%$ concentration of Sigmacell was used in these measurements.

cycles. In terms of accuracy, the second Lagrangian algorithm had the smallest variances, and the scaling factor estimation resulted in the largest ones (see Table I).

The influence of the concentration of acoustic scatterers on Young's moduli was investigated on eight PVA samples. All samples underwent two freeze-thaw cycles, and the Sigmacell concentration was varied from $1 \%$ to $4 \%$. Overall, as shown in Fig. 9, a mean increase of the Young's modulus by $54 \mathrm{kPa}$ was observed as the concentration was increased. More specifically, the mean Young's modulus computed for the four elastography methods and the stretching test was $91 \mathrm{kPa}$ for $1 \%$ of Sigmacell. It was $104 \mathrm{kPa}, 141 \mathrm{kPa}$, and $145 \mathrm{kPa}$ for concentrations of $2 \%, 3 \%$, and $4 \%$, respectively.

\section{Discussion}

It is important to notice that the viscoelasticity of PVA was neglected in this study, first because the relaxation

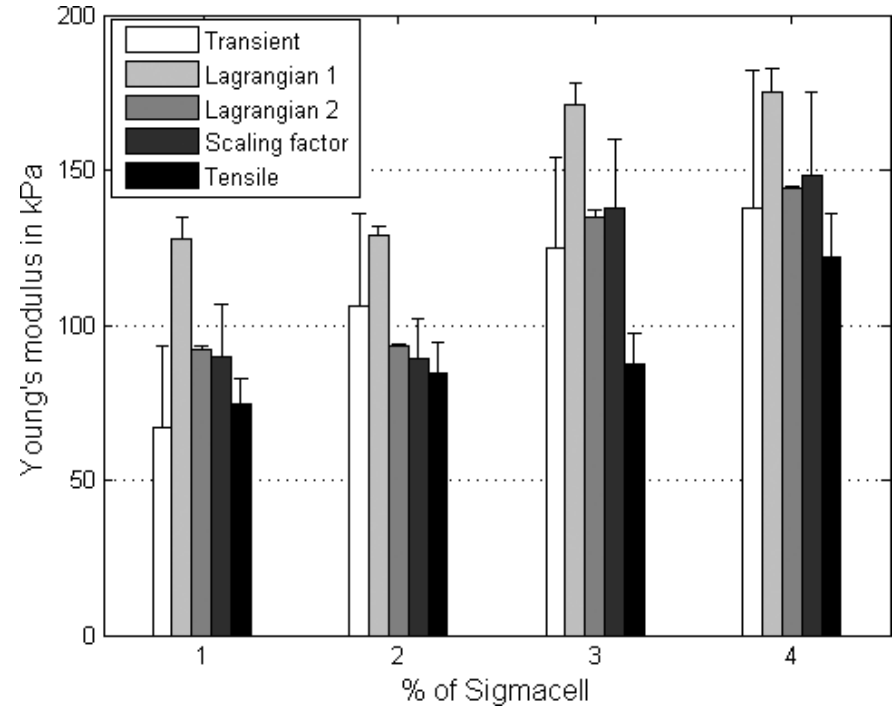

Fig. 9. Young's moduli as a function of the ratio of Sigmacell acoustic scatterers added in the phantoms. The number of freeze-thaw cycles was fixed to two for those measurements.

time of PVA is long [2]. Consequently, the different loading frequencies - used for the transient, quasistatic and mechanical tests - had all characteristic time smaller than the relaxation time. Second, to reduce the viscoelastic effects, samples underwent preload cycles until the stressstrain curve became stable, which happened as soon as the second preload cycle. As the visco-elasticity is small, the Young moduli measured with the transient elastography and other quasistatic methods were expected to be the same.

A first remark concerns the estimated Young's moduli that could be varied in a large range, from $20 \mathrm{kPa}$ to $600 \mathrm{kPa}$, approximately (see Fig. 8). This is satisfying if one's interest is to model biological soft tissues. For instance, Young's moduli were reported to vary in such a range for human tissues [36] (28 $\mathrm{kPa}$ for the normal glandular tissue of breast [37] to $630 \mathrm{kPa}$ for the harder, healthy, common carotid artery [38]). A second remark concerns the reproducibility of the phantom building procedure, which, according to Fig. 7, looks acceptable with 
TABLE II

Covariance Between the Stretching Tests and Elastographic Methods.

\begin{tabular}{lccc}
\hline Method & Covariance & Offset & $\mathrm{R}^{2}$ \\
\hline Transient & 1.02 & 14 & 0.93 \\
Lagrangian 1 & 1.07 & 13.8 & 0.99 \\
Lagrangian 2 & 1.02 & -3.6 & 0.99 \\
Scaling factor & 0.97 & -1.4 & 0.98 \\
\hline
\end{tabular}

the bias of the three samples with the same number of freeze-thaw cycles smaller than the gold standard accuracy. The only exception is for seven cycles in which two samples were very different from the mean value; this is attributed to measurement uncertainty of a single sample estimated with a very high Young's modulus $(313 \mathrm{kPa}$, $371 \mathrm{kPa}, 511 \mathrm{kPa}$ ), which artificially increased the mean value. The errors came from two sources, the precision of the test instrument and the possible irregular geometry of the phantoms.

Concerning the elastography methods, the algorithms gave consistently similar mean Young's moduli to those measured with the gold standard tensile instrument. However, between one to six freeze-thaw cycles, either the transient elastography or the first Lagrangian method, gave the worst matching with the gold standard method. The second Lagrangian and time-scaling factor methods looked excellent for one freeze-thaw cycle. However, the best estimation was obtained with the second Lagrangian estimator for two and three freeze-thaw cycles. For four cycles, the first Lagrangian estimator was the best. The transient elastography and time-scaling factor methods provided the closest estimations to the gold standard for five and six cycles. For PVA-C samples with a higher number of freezethaw cycles, the consistency of the results declined. To further compare the different methods, the correlation between the mean Young's moduli obtained with the stretching test and those estimated with the elastography methods was measured on samples that underwent from one to six cycles (see Table II). The covariance, the offset, and the regression coefficient are reported on Table II. It can be concluded that the different elastography methods were consistent with a covariance close to one and correlation coefficients above 0.93 .

A few reasons can explain the worse results between 7 to 10 freeze-thaw cycles. We first had difficulties in cutting symmetric samples due to their rigidity at these numbers of cycles. In addition, during the fabrication process of PVA-C, it was noticed that samples with a high number of freeze-thaw cycles had a tendency to dehydrate. Consequently, a layer of ice appeared between the sample and the mould. This layer became noticeable for samples corresponding to a number of cycles for which the density of PVA began to stabilize [see Fig. 3(a)]. It is known that some macropores of solvent, water in that case, remain in the hydrogel samples after several freeze-thaw cycles [3]. A possible explanation for the logarithmic increasing rela- tionship of the curve expressing the density of the samples as a function of the number of cycles can be that, after six cycles, a large part of the macropores containing the solvent had disappeared, leaving only the polymer network. The Young's moduli calculated with transient elastography also were less reliable. An explanation is that, when samples became stiffer and because the derivation window sizes were maintained constant for the whole experiment, the shear wave speed was more noisy as the number of wavelengths decreased.

Except for the number of freeze-thaw cycles, the most important parameter to control during the building process was the freezing-thawing rate. In a previous work [39], a very different mean Young's modulus was measured for a 10\% PVA solution and a 24-hour freeze-thaw cycle. The only differences with respect to the current study was the use of a colder freezing temperature of $-40^{\circ} \mathrm{C}$, shorter freezing and thawing rates, and a $1 \%$ concentration of acoustic scatterers. In [39], the rates of change of the temperature were not regulated, samples were directly introduced in the freezer and put out of it to room temperature during thawing. The difference between both studies thus emphasizes the important role of the freeze-thaw temperature variations in the cross-linking process of PVA. As an example, the Young's modulus measured for a five-cycles phantom was $90 \pm 6 \mathrm{kPa}$ in [39], and it was $300 \pm 35 \mathrm{kPa}$ for the current study. As the smallest Young modulus found in the current study was about $25 \mathrm{kPa}$, which is above values corresponding to some biological tissues, increasing the speed rate could be a good method to create samples with lower Young's moduli. Another alternative could be to use PVA solution at a lower concentration [1].

Different elastography methods were tested and compared to gold standard assessments. The stretching test has been chosen as the gold standard because it is less sensitive to geometrical artifacts. Transient elastography has a big advantage in that it does not depend on the boundary conditions, provides directly the Young's modulus, and allows one to estimate the Poisson's ratio. However, a large-size phantom is necessary for measurements. The bigger the medium, the more precise the estimation, because more shear wavelengths propagate. Another limit of this last method is that the harder the samples, the higher the shear wave speeds and the more difficult it becomes to differentiate the shear wave from the bulk wave. Quasistatic elastography has the advantage of providing a whole image of strain distribution. Furthermore, Lagrangian methods also estimate 2-D deformations, including shears, and the scaling factor algorithm advantage is the shorter computational time. As the quasistatic methods were used with a compression setup, their estimated strain images are strongly dependent on the geometry. When the surface of samples are not flat, as was the case for the high number of freeze-thaw cycles, the stress within samples is not uniform and, consequently, the estimated strain also varies.

Another aspect is the estimation of Poisson's ratio with good precision thanks to the measurement of shear speed. 
The estimated Poisson's ratios were $0.499 \pm 0.001$ and definitely shows that PVA cryogel phantoms are incompressible.

\section{CONCLusions}

Four elastography methods have been evaluated on tissue-mimicking phantoms. The Young's modulus estimated via the inverse problem of each method was compared to the elastic modulus measured with a mechanical test instrument. The different elastographic methods showed good agreement with the mechanical tests, for a number of freeze-thaw cycles between one and six, thanks to the flat geometry of the samples. Correlations above 0.93 were observed between the elastography methods and the mechanical stretching test. For more than six freezethaw cycles, the sample geometry became more difficult to control, and the results were less reliable.

A rigorous characterization of PVA cryogel also was done. A precise building procedure was described, and different operators were involved in the study to test reproducibility. Acoustic (speed of sound, shear wave speed) and mechanical (Young's modulus, Poisson's ratio, density) properties were evaluated. The influence of the number of cycles and of the quantity of acoustic scatterers was assessed. Thanks to shear wave measurements, an estimation of the Poisson's ratios was provided. Mean values of $0.499 \pm 0.001$ proved the incompressibility of the material. Young's moduli of the samples varied from $20 \mathrm{kPa}$, a typical value of soft tissues such as breast or liver, to $600 \mathrm{kPa}$, a typical value for harder tissues such as arteries, were obtained in this study, which validate the PVA as a good tissue-mimicking material.

\section{ACKNowledgments}

The authors want to thank Nusrat Choudhury, Richard Leask, Amar Amararene, and Boris Chayer for some illuminating discussions and technical assistance.

\section{REFERENCES}

[1] C. M. Hassan and N. A. Peppas, "Structure and applications of poly(vinyl alcohol) hydrogels produced by conventional crosslinking or by freezing/thawing methods," Adv. Polymer Sci., vol. 153, pp. 37-65, 2000.

[2] W. K. Wan, G. Campbell, Z. F. Zhang, A. J. Hui, and D. R. Boughner, "Optimizing the tensile properties of polyvinyl alcohol hydrogel for the construction of a bioprosthetic heart valve stent," J. Biol. Mater. Res., vol. 63, no. 6, pp. 854-861, 2002.

[3] V. I. Lozinsky, "Cryotropic gelation of poly(vinyl alcohol) solutions," Rus. Che. Rev., vol. 67, no. 7, pp. 573-586, 1998.

[4] V. I. Lozinsky, "Cryogels on the basis of natural and synthetic polymers: Preparation, properties and application," Uspekhi Khimii, vol. 71, no. 6, pp. 559-585, 2002.

[5] T. J. Hall, M. Bilgen, M. F. Insana, and T. A. Krouskop, "Phantom materials for elastography," IEEE Trans. Ultrason., Ferroelect., Freq. Contr., vol. 44, no. 6, pp. 1355-1365, 1997.
[6] E. L. Madsen, M. A. Hobson, H. Shi, T. Varghese, and G. R. Frank, "Tissue-mimicking agar/gelatin materials for use in heterogeneous elastography phantoms," Phys. Med. Biol., vol. 50, pp. 5597-5618, 2005.

[7] W. D. D’Souza, E. L. Madsen, O. Unal, K. K. Vigen, G. R. Frank, and B. R. Thomadsen, "Tissue mimicking materials for a multi-imaging modality prostate phantom," Med. Phys., vol. 28, no. 4, pp. 688-700, 2001.

[8] L. K. Ryan and F. S. Foster, "Tissue equivalent vessel phantoms for intravascular ultrasound," Ultrasound Med. Biol., vol. 23, no. 2, pp. 261-273, 1997.

[9] R. J. Dickinson and C. R. Hill, "Measurement of soft tissue motion using correlation between A-scans," Ultrasound Med. Biol., vol. 8, no. 3, pp. 263-271, 1982.

[10] J. Ophir, I. Cespedes, H. Ponnekanti, Y. Yazdi, and X. Li, "Elastography - A quantitative method for imaging the elasticity of biological tissues," Ultrason. Imag., vol. 13, no. 2, pp. 111-134, 1991.

[11] R. L. Maurice, J. Ohayon, G. Finet, and G. Cloutier, "Adapting the Lagrangian speckle model estimator for endovascular elastography: Theory and validation with simulated radio-frequency data," J. Acoust. Soc. Amer., vol. 116, no. 2, pp. 1276-1286, 2004.

[12] R. L. Maurice and M. R. Bertrand, "Lagrangian speckle model and tissue-motion estimation-Theory," IEEE Trans. Med. Imag., vol. 18, no. 7, pp. 593-603, 1999.

[13] R. L. Maurice, J. Ohayon, Y. Fretigny, M. R. Bertrand, G. Soulez, and G. Cloutier, "Noninvasive vascular elastography: Theoretical framework," IEEE Trans. Med. Imag., vol. 23, no. 2, pp. 164-180, 2004.

[14] E. E. Konofagou, T. Varghese, and J. Ophir, "Spectral estimators in elastography," Ultrasonics, vol. 38 , no. 1-8, pp. 412-416, 2000.

[15] T. Varghese, E. E. Konofagou, J. Ophir, S. K. Alam, and M. Bilgen, "Direct strain estimation in elastography using spectral cross-correlation," Ultrasound Med. Biol., vol. 26, no. 9, pp. 1525-1537, 2000.

[16] S. Srinivasan and J. Ophir, "A zero-crossing strain estimator for elastography," Ultrasound Med. Biol., vol. 29, no. 2, pp. 227238, 2003.

[17] M. O'Donnell, A. R. Skovoroda, B. M. Shapo, and S. Y. Emelianov, "Internal displacement and strain imaging using ultrasonic speckle tracking," IEEE Trans. Ultrason., Ferroelect., Freq. Contr., vol. 41, no. 3, pp. 314-325, 1994.

[18] C. L. de Korte, I. Cespedes, A. F. van der Steen, G. Pasterkamp, and N. Bom, "Intravascular ultrasound elastography: Assessment and imaging of elastic properties of diseased arteries and vulnerable plaque," Eur. J. Ultrasound, vol. 7, no. 3, pp. 219224, 1998.

[19] A. Pesavento, C. Perrey, M. Krueger, and H. Ermert, "A time efficient and accurate strain estimation concept for ultrasonic elastography using iterative phase zero estimation," IEEE Trans. Ultrason., Ferroelect., Freq. Contr., vol. 45, no. 5, pp. 10571066, 1999.

[20] S. K. Alam, J. Ophir, and E. E. Konofagou, "An adaptive strain estimator for elastography," IEEE Trans. Ultrason., Ferroelect., Freq. Contr., vol. 45, no. 2, pp. 461-472, 1998.

[21] E. Brusseau, C. Perrey, P. Delachartre, M. Vogt, D. Vray, and H. Ermert, "Axial strain imaging using a local estimation of the scaling factor from RF ultrasound signals," Ultrason. Imag., vol. 22, no. 2, pp. 95-107, 2000.

[22] S. Catheline, F. Wu, and M. Fink, "A solution to diffraction biases in sonoelasticity: The acoustic impulse technique," $J$. Acoust. Soc. Amer., vol. 105, no. 5, pp. 2941-2950, 1999.

[23] J.-L. Gennisson, S. Catheline, S. Chaffaï, and M. Fink, "Transient elastography in anisotropic medium: Application to the measurement of slow and fast shear waves velocities in muscles," J. Acoust. Soc. Amer., vol. 114, no. 1, pp. 536-541, 2003.

[24] L. Sandrin, M. Tanter, J.-L. Gennisson, S. Catheline, and M. Fink, "Shear elasticity probe for soft tissues with 1-D transient elastography," IEEE Trans. Ultrason., Ferroelect., Freq. Contr., vol. 49, no. 4, pp. 436-446, 2002.

[25] J. Fromageau, H. Liebgott, E. Brusseau, D. Vray, and P. Delachartre, "Estimation of time-scaling factor for ultrasound medical imaging using the Hilbert transform," Eur. Assoc. Signal Process., J. Adv. Signal Process., 2007, to be published. 
[26] R. L. Maurice, E. Brusseau, G. Finet, and G. Cloutier, "On the potential of the Lagrangian speckle model estimator to characterize atherosclerotic plaques in endovascular elastography: In vitro experiments using an excised human carotid artery," Ultrasound Med. Biol., vol. 31, no. 1, pp. 85-91, 2005.

[27] C. Schmitt, "Non invasive vascular elastography: Toward a complementary characterization tool of atherosclerosis in carotid arteries," M.S. thesis, University of Montréal, Montréal, QC, Canada, Jun. 2005. (in French)

[28] H. Du, J. Liu, C. Pellot-Barakat, and M. F. Insana, "Noise minimization by multi-compression approach in elasticity imaging," in Proc. IEEE Ultrason. Symp., 2004, pp. 32-35.

[29] R. L. Maurice, M. Daronat, J. Ohayon, E. Stoyanova, F. S. Foster, and G. Cloutier, "Non-invasive high-frequency vascular ultrasound elastography," Phys. Med. Biol., vol. 50, pp. 1611$1628,2005$.

[30] S. Periaswamy and H. Farid, "Elastic registration in the presence of intensity variations," IEEE Trans. Med. Imag., vol. 22, no. 7, pp. 865-874, 2003.

[31] S. Catheline, F. Wu, and M. Fink, "Diffraction field of a low frequency vibrator in soft tissues using transient elastography," IEEE Trans. Ultrason., Ferroelect., Freq. Contr., vol. 46, no. 4, pp. 1013-1020, 1999.

[32] D. Royer and E. Dieulesaint, Elastic Waves in Solid. vol. 1, Berlin: Springer-Verlag, 1996.

[33] L. D. Landau and E. M. Lifshitz, Theory of Elasticity. London: Butterworth-Heineman, 2002.

[34] L. Li, E. Beniash, E. Zubarev, W. Xiang, R. Rabatic, G. Zhang, and S. Stupp, "Assembling a lasing hybrid material with supramolecular polymers and nanocrystals," Nature Mater., vol. 2, pp. 689-694, 2003.

[35] T. Elshazly, "Characterization of PVA hydrogels with regards to vascular graft development," M.S. thesis, Georgia Institute of Technology, Atlanta, GA, May 2004.

[36] A. P. Sarvazyan, A. R. Skovoroda, S. Y. Emelianov, J. B. Fowlkes, J. G. Pipe, R. S. Adler, R. B. Buxton, and P. L. Carson, "Biophysical bases of elasticity imaging," Acoust. Imaging, vol. 21, pp. 223-240, 1995.

[37] T. Krouskop, T. Wheeler, F. Kallel, B. S. Garra, and T. Hall, "Elastic moduli of breast and prostate tissues under compression," Ultrason. Imag., vol. 20, pp. 260-274, 1998.

[38] R. H. Selzer, M. J. Mack, P. L. Lee, H. Kwon-Fu, and H. N. Hodis, "Improved common carotid elasticity and intima-media thickness measurements from computer analysis of sequential ultrasound frames," Atherosclerosis, vol. 154, no. 1, pp. 185193, 2001.

[39] J. Fromageau, E. Brusseau, D. Vray, G. Gimenez, and P. Delachartre, "Characterization of PVA cryogel for intravascular ultrasound elasticity imaging," IEEE Trans. Ultrason., Ferroelect., Freq. Contr., vol. 50, no. 10, pp. 1318-1324, 2003.

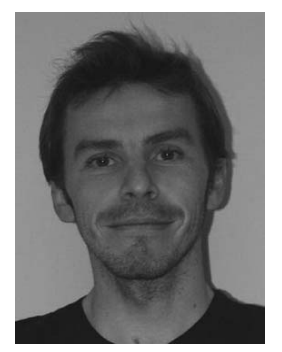

Jérémie Fromageau was born in Orléans, France, in 1975. He received the M.S. degree in physical acoustics from the University Denis Diderot, Paris 7, in 1999, and the Ph.D. degree in medical image processing from the Institut National des Sciences Appliquées (INSA), Lyon, France, in 2003.

He is currently a research associate in the Laboratory of Biorheology and Medical Ultrasonics, University of Montreal Hospital Research Center, Montreal, Quebec, Canada.

His research interests include signal and image processing applied to medical ultrasound imaging, elastography, and high-frequency imaging.

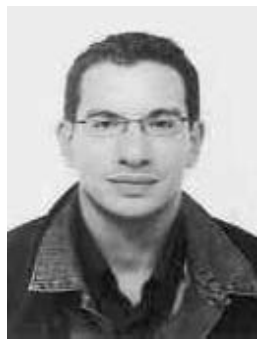

Jean-Luc Gennisson was born in 1974 in France. He received the DEA degree in electronics in 2000 from the University of Paris VI. In 2003, he received the Ph.D. degree in physics (acoustics) from the University of Paris VI for his work on ultrasound elastography. From 2003 to 2005, he worked at the Laboratory of Biorheology and Medical Ultrasonics in Montreal, Montreal, Quebec, Canada, for a postdoctoral fellowship with Dr. Guy Cloutier. In 2005, he became a research sci(CNRS), Paris, France. entist of the French National Research Center

His current research interests include medical ultrasonic imaging, shear wave propagation in soft tissues for cancer detection, and nonlinear shear waves.

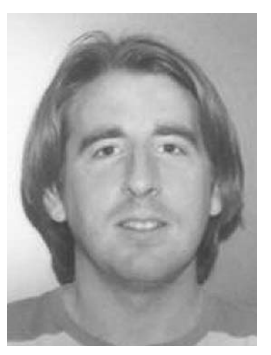

Cédric Schmitt was born in September 1978 in Hyères, France. He graduated in 2002 from the École Nationale Supérieure des Ingénieurs Électriciens de Grenoble (part of the Institut National Polytechnique de Grenoble), Grenoble, France, majoring in signal processing and systems. In 2003, he joined the Laboratory of Biorheology and Medical Ultrasonics, University of Montreal Hospital Research Center, Montreal, Quebec, Canada, where he received his M.Sc. degree in biomedical engineering from the Institute of Biomedical Engineering of the University of Montreal in 2005. His research was focusing on the characterization of atherosclerosis in carotid arteries by using noninvasive, vascular elastography methods. He is currently working toward a Ph.D. degree in biomedical engineering at the University of Montreal.

His research interests are in biomedical image and signal processing, experimental ultrasonography, vascular imaging, characterization of carotid atherosclerosis, static and dynamic elastography, and vascular phantom designs.

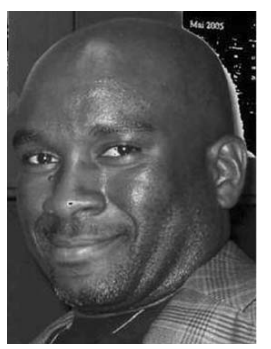

Roch L. Maurice received his Ph.D. degree in biomedical engineering from the University of Montreal, Montreal, Quebec, Canada, in 1998. He is currently a scientist at the University of Montreal Hospital Research Center and a research assistant professor in the Department of Radiology, Radio-Oncology and Nuclear Medicine of the University of Montreal.

His major interests are mathematical modeling for the purpose of characterizing soft biological tissues with specific applications in cardiovascular and breast cancer fields using ultrasound.

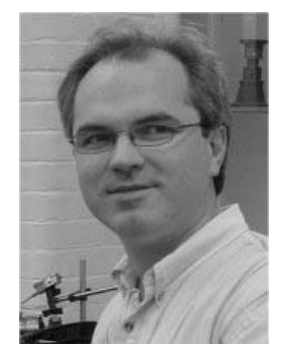

Rosaire Mongrain is an associate professor of mechanical engineering at McGill University, Montreal, Quebec, Canada.

His principal research activities include the design optimization of blood-interacting devices (interventional catheters, ventricular assist devices, heart valves, stents, grafts, filters), blood-flow modeling with heat, and mass transfer in interaction with cardiovascular devices. Recent works include the study of atherosclerotic plaque rupture, aortic valve dynamics, and diffusion kinetics of drugeluting stents. 


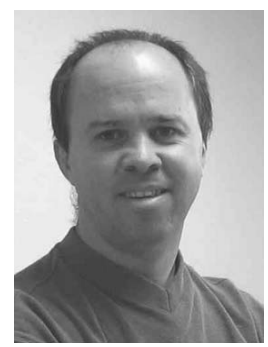

Guy Cloutier (S'89-M'90) obtained his B.Eng. degree in electrical engineering from the Université du Québec à Trois-Rivières, Trois-Rivières, QC, Canada, in 1983, and his M.Sc. and Ph.D. degrees in biomedical engineering from the École Polytechnique de Montréal in 1986 and 1990, respectively. Between 1990 and 1992, he pursued a postdoctoral training with Prof. K. Kirk Shung at the Laboratory of Medical Ultrasonics, Bioengineering Program, The Pennsylvania State University, University Park, PA.

Dr. Cloutier is currently Director of the Laboratory of Biorheology and Medical Ultrasonics at the Research Center of the University of Montreal Hospital, Montreal, Quebec, Canada, Member of the Institute of Biomedical Engineering of the University of Montreal, and Professor in the Department of Radiology, Radio-Oncology and $\mathrm{Nu}-$ clear Medicine of the University of Montreal.
His research interests are the characterization of red blood cell aggregation dynamics with ultrasound and rheological methods, the development of small animal imaging methods to study blood flow disorders, the characterization of biomechanical properties of vascular wall structures with ultrasound elastography, the 3-D morphologic and hemodynamic assessment of lower limb arterial stenoses, and mathematical and biomechanical modeling.

He has published more than 75 peer-reviewed papers and book chapters in these fields; he holds four patents (pending); he is a member of the Advisory Editorial Board for the journals Ultrasound in Medicine and Biology and Current Medical Imaging Reviews; and he served on several grant review study sections of the Heart and Stroke Foundation of Canada, the Canadian Institutes of Health Research, the Fonds de la Recherche en Santé du Québec (FRSQ), the Canada Research Chairs, and the National Institutes of Health of the United States. Dr. Cloutier is the recipient of a National Scientist award from FRSQ, 2004-2009. 